\title{
TERAPIA OCUPACIONAL Y EDUCACIÓN INCLUSIVA: ASPECTOS RELACIONADOS AL DESEMPEÑO OCUPACIONAL DE PERSONAS CON DISCAPACIDAD
}

\author{
OCCUPATIONAL THERAPY AND INCLUSIVE EDUCATION: ASPECTS RELATED TO \\ OCCUPATIONAL PERFORMANCE OF PEOPLE WITH DISABILITIES
}

\section{Ivanilda Costa da Rosa ${ }^{1}$, Beatriz Angélica Valdivia Arancibia ${ }^{2}$, Franciele Cascaes da Silva ${ }^{3}$, Paulo José Barbosa Gutierres Filho ${ }^{4}$, Rudney da Silva ${ }^{5}$.}

\begin{abstract}
Resumen:
La Educación inclusiva busca la igualdad de oportunidades para todos, y la Terapia Ocupacional puede contribuir para este proceso por medio del desarrollo del desempeño ocupacional. En este sentido se busca analizar las contribuciones de la Terapia Ocupacional para la educación inclusiva mediante los aspectos relacionados al desempeño ocupacional de personas con discapacidad. Por lo tanto, este estudio se caracterizó como una revisión que incluye obras de literatura especializada sobre la educación inclusiva, Terapia Ocupacional y desempeño ocupacional, destacando la Medida Canadiense de desempeño Ocupacional (COPM), que es fundamentada en las directrices de la Terapia Ocupacional para la Práctica Basada en el Cliente. A pesar de la carencia de estudios sobre este tema, fueron seleccionados artículos completos, publicados en la última década en la lengua inglesa y portuguesa. Se puede concluir que la COPM permite identificar las principales actividades problemas, proporcionando subsidios para mejorar el desempeño y lograr satisfacción en las actividades seleccionadas. La actuación del terapeuta ocupacional en interacción con las personas con discapacidad en la educación inclusiva, puede contribuir significativamente para el desarrollo satisfactorio del rol ocupacional, promoviendo un desempeño ocupacional competente, favoreciendo una amplia gama de posibilidades ocupacionales.
\end{abstract}

\section{Palabras clave:}

Educación inclusiva, Terapia ocupacional, Medida Canadiense de Desempeño Ocupacional.

1 Terapeuta Ocupacional. Especialista. Investigador (a). Universidad del Estado de Santa Catarina, Centro de Ciencias de la Salud y do Deporte, Calle Pascoal Simone, 358 - Coqueiros - Florianópolis, SC, Brasil. CEP: 88080-350. Fone: 55 (48) 3321 8651. rosaivanilda@ig.com.br.

2 Educadora Física. Especialista. Pesquisadora. Universidad del Estado de Santa Catarina, Centro de Ciencias de la Salud y do Deporte, Calle Pascoal Simone, 358 - Coqueiros - Florianópolis, SC, Brasil. CEP: 88080-350. Fone: 55 (48) 3321 8651. beangelval@hotmail.com.

3 Fisioterapeuta. Especialista. Pesquisadora. Universidad del Estado de Santa Catarina, Centro de Ciencias de la Salud y do Deporte, Calle Pascoal Simone, 358 - Coqueiros - Florianópolis, SC, Brasil. CEP: 88080-350. Fone: 55 (48) 3321 8651. francascaes@yahoo.com.br.

4 Fisioterapeuta. Doctor. Pesquisador y Profesor. Universidad del Estado de Santa Catarina, Centro de Ciencias de la Salud y do Deporte, Calle Pascoal Simone, 358 - Coqueiros - Florianópolis, SC, Brasil. CEP: 88080-350. Fone: 55 (48) 3321 8651. paulogutierresfilho@ig.com.br.

5 Educador Físico. Doctor. Pesquisador y Profesor. Universidad del Estado de Santa Catarina, Centro de Ciencias de la Salud y do Deporte, Calle Pascoal Simone, 358 - Coqueiros - Florianópolis, SC, Brasil. CEP: 88080-350. Fone: 55 (48) 3321 8651. rudney.silva@udesc.br. 


\begin{abstract}
:
Inclusive education seeks equality of opportunity for all and Occupational Therapy can contribute to this process through the development of occupational performance. In this regard, we attempted to analyze the Occupational Therapy contributions for inclusive education through of the aspects related to occupational performance of the people with disabilities. Therefore, this study was characterized as a review that included works of literature on inclusive education, occupational therapy and occupational performance, giving prominence to the Canadian Occupational Performance Measure (COPM), which is based on the Guidelines of Occupational Therapy Practice-Based Client. We selected yet, despite the lack of studies, articles published in the last decade in English and Portuguese. It can be concluded that the COPM identifies the main activities problems and offer suggestions for improving the performance and satisfaction of selected activities. The role of the occupational therapist with people with disabilities in inclusive education can contribute significantly to the development of satisfactory occupational roles, promoting a competent occupational performance favoring the discovery of new occupational opportunities.
\end{abstract}

\title{
Keywords:
}

Inclusive education, Occupational therapy, Canadian Occupational Performance Measure.

\section{INTRODUCCIÓN}

La propuesta de la educación inclusiva es una educación de calidad para todos, en la cual las personas excluidas y la sociedad, pretenden alcanzar la igualdad de oportunidades, porque las modificaciones del eje principal están en las escuelas; y éstas, deberán reestructurarse para atender a toda la diversidad humana existente (Mendes eg. 2002, 2006). En relación a los recursos humanos, se torna imprescindible la inserción y el soporte de profesionales del área de la salud en el ámbito de la educación inclusiva. En este sentido, la función del terapeuta ocupacional se ha utilizado más como una extensión de la atención ofrecida en servicios de rehabilitación. En tanto que en Brasil la relación entre la educación y la terapia ocupacional comenzó por medio de la intervención directa de la educación especial, específicamente en escuelas y clases especiales dirigidas a poblaciones específicas (Rocha EF., 2007).

Con la llegada de la educación inclusiva, el terapeuta ocupacional tuvo que re direccionar su contexto y su forma de trabajo desde un enfoque netamente de rehabilitación, y avanzar para una rehabilitación educativa e inclusiva, ya que la actuación de este profesional es cada vez más constante en la educación, con el fin de comprender y contribuir a la producción teórica, discutiendo sobre cómo esta práctica se ajusta a los objetivos de la educación inclusiva (Lorenço GF., 2010).

De este modo, varias son las actividades posibles en el área de la terapia ocupacional, desde la práctica te- rapéutica que propone un espectro de posibilidades en este campo de actuación, promoviendo transformaciones y produciendo valores, auxiliar en el trabajo de organización y cuidados diarios, hasta las oportunidades de construcción de autonomía e independencia, generando autoconfianza y autoestima (Bartalotti, 2001).

La terapia ocupacional en el área de la educación viene a facilitar la comprensión del proceso de enseñanza-aprendizaje, que está estrechamente relacionado al conocimiento sobre el desarrollo humano y su desempeño ocupacional en tareas rutinarias en el contexto educacional (Rocha EF., Luiz A., Zulian MA., 2003)

Una de las formas de evaluación del desempeño ocupacional, es a través de la Medida Canadiense de Desempeño Ocupacional (Canadian Measure of Occupational Performance) - COPM, considerado uno de los principales instrumentos de evaluación del desempeño ocupacional y utilizado mundialmente por terapeutas ocupacionales, cuyo objetivo apunta a colaborar en la evaluación de los efectos de la práctica de los terapeutas ocupacionales en desempeño ocupacional de las personas, fundamentada teóricamente en la práctica clínica centrada en el cliente (CAOT, 1991). Considerando la práctica clínica, Parker y Sykes (2006), apunta que uno de los aspectos positivos de la COMP es su sensibilidad para medir el resultado de una variedad de intervenciones de la terapia ocupacional, más allá del hecho de que este instrumento puede servir de utilidad para personas de diferentes entornos, así como las discapacidades incapacidades, especialmente por ser una medida independiente de diagnósticos específicos. 
Estos temas reafirman que la COPM haya sido traducida en más de veinte idiomas, siendo utilizada en treinta y cinco países y adaptada a los más diversos contextos, como puede ser observado en la extensa bibliografía sobre éste (Rebeiro, 2000; Carpenter y col., 2001; Sumsion 2003. Carswell y col., 2004).

Oliveira y Castanharo (2008) demostraron a través de una revisión bibliográfica, la importancia de la intervención de la terapia ocupacional en el contexto escolar con niños que padecen disturbios y trastornos de aprendizaje, y propusieron la Medida Canadiense de Desempeño Ocupacional como sustento para la intervención, porque se verificó que los educadores, padres responsables y los propios alumnos son los principales agentes de esta propuesta, que comprometidos en el desarrollo del proceso de la educación inclusiva, confirman que el terapeuta ocupacional puede intervenir actuando como facilitador, y así generar una mejor interacción entre el alumno, sus profesores y sus familias; y mejorar el bienestar emocional del niño o niña, ya que en la mayoría de los casos es motivo de exclusión en este contexto.

Law (2009) apunta que la expectativa de la traducción del manual y del protocolo de entrevista pueda incentivar el desarrollo de investigaciones que confirmen las propiedades psicométricas y la utilidad clínica del instrumento en la cultura brasileña, y que la COPM sea incorporada como un recurso adicional en la evaluación de las personas. Asimismo, que pueda ser usada en investigaciones mostrando la eficacia en los procedimientos de intervención utilizados por terapeutas ocupacionales en las diferentes áreas de desempeño.

De este modo, este estudio tiene como objetivo analizar las contribuciones de la Terapia Ocupacional en la educación inclusiva, mediante los aspectos relacionados al desempeño ocupacional de personas con discapacidad.

Para esto, fueron realizadas técnicas fundamentales de la revisión sistemática, procedimientos profundos y extensos de la literatura. Fueron sistemáticamente revisadas las bases de datos Web of Science, Journals@ ovid, Science Direct, Scopus, SportDiscus y Scientific Eletronic Library Online. La búsqueda incluyó el descriptor "occupational performance" (desempeño ocupacional) en idioma español y portugués, disponible en las palabras clave de los artículos. También se realizó una búsqueda manual de obras de referencias en los artículos identificados en la revisión sistemática.

Como fue verificada una escasez de artículos sobre la temática en cuestión, no fueron adoptados paráme- tros de tiempo de publicación y tampoco de tipo de obra. Se realizaron también levantamientos en la literatura especializada de las ciencias de la ocupación, ciencias de la rehabilitación y ciencias del movimiento humano, para la revisión bibliográfica. Los procedimientos de identificación y selección de obras, fueron orientados por directrices de gran relevancia referentes a los autores y las obras, en especial, de libros de referencia y manuales. En ambos casos, fueron utilizadas técnicas básicas de análisis de contenido. Después de la sistematización de los materiales, el análisis teórico fue organizado en tres ítems: a) Educación Inclusiva; b) Terapia Ocupacional; y c) Desempeño Ocupacional.

\section{Educación Inclusiva}

Como consecuencia de un movimiento mundial bastante amplio denominado Inclusión Social, la Educación Inclusiva ganó renombre en Brasil desde finales de la década de 1980 con la promulgación de la Constitución Federal, que estableció que la educación es un derecho de todos, que se direcciona frente al movimiento internacional sobre el derecho de todos a la educación de calidad, y principalmente, a la construcción de un proceso en el cual las personas excluidas y la sociedad buscan equiparar las oportunidades para todos (Constitución de Brasilia,1998; Mendes, 2002). En este sentido, Mantoan (1998) afirma que para incluir a todas las personas, la sociedad debe ser modificada a partir de la comprensión de que ésta necesita ser capaz de atender las necesidades de sus miembros, lo que hace que la meta de la inclusión sea dejar a nadie fuera del sistema escolar, debiendo adaptarse a las particularidades de todas las personas.

En Brasil, según Santos (2000), una serie de leyes federales, estaduales y municipales fueron promulgadas para hacer efectivo el compromiso asumido en la Declaración de Salamanca, teniendo en cuenta que "las escuelas deben acoger a todos los niños independientemente de sus condiciones físicas, sociales, psicológicas, de lenguaje, entre otras". Entre estas propuestas, se encuentra la Ley de Directrices y Bases de la Educación Nacional (LDB) de 1996, que busca promover el derecho de la infancia y de la adolescencia en relación a la educación, destacando la importancia en la atención de los estudiantes con discapacidad en el sistema de educación. (Ley Brasil, 1996; BARTALOTTI CC., A/B MMRP., 2001). 
Chalita (2004), considera a todas las personas diferentes, ya que cada ser humano es único. El mayor logro de la Constitución de 1988 fue colocar en un mismo espacio las desigualdades, una educación que facilita que las desigualdades convivan en un mismo ambiente y aprendan el ejercicio del compañerismo, desenvolviendo la capacidad de colaboración y ayuda mutua para la superación de obstáculos. Así, Almeida y Campos (2002), describen que estos logros no impedirán el avance de los procesos sociales de exclusión, que en sentido opuesto, profundizarán las contradicciones en la sociabilidad de este segmento. Si por un lado la legislación garantiza la participación en la producción y repartición de los bienes, servicios y valores sociales; por otro lado, tal como ocurrió con otros segmentos sociales, se verificó la ausencia de políticas que sustenten el verdadero ejercicio de estos derechos.

Costabile y Brunello (2005), en un estudio, reflexionaron cualitativamente sobre los impactos de la educación inclusiva en la vida diaria de niños con discapacidad, a partir de entrevistas abiertas con las familias de los niños con discapacidad, insertados en clases regulares de las escuelas públicas. Constataron a través de investigaciones que la legislación facilita el ingreso de niños con discapacidad en las escuelas regulares del sistema educativo público de enseñanza, pero muchas veces no se garantiza la inclusión escolar, por la falta de condiciones satisfactorias para el aprendizaje de los niños, que se justifica en el número elevado de estudiantes en la sala de clases, la falta de apoyo de los profesores, la poca información del equipo técnico educacional, la falta de preparación a nivel de las escuelas y la falta de políticas que apoyen estos trabajos.

Correa (2001) afirma que las escuelas no están preparadas para trabajar adecuadamente con las dificultades y diferencias de aprendizaje que los estudiantes presentan a lo largo de su etapa escolar. Para este autor, el no aprender no está relacionado solamente con el fracaso escolar (en términos de reprobación, repitencia o bajo rendimiento en pruebas), sino que también, con la ausencia de progresos en el proceso de aprendizaje. Rocha, Luiz y Zulian (2003), enfatizan que el profesor preparado para la inclusión debería estar capacitado para diferentes estrategias pedagógicas, posibles de ser aplicadas con diferentes estudiantes, independientemente de si tienen discapacidad o no. Mantoan (2006) agrega que la concretización de la inclusión, exige no solamente políticas públicas eficaces, sino también, una reorganización de toda la comunidad para la aceptación de las diferencias, lo que impone una mudan- za de perspectiva educacional, ya que no se limita a los que presentan discapacidades, porque se extiende a cualquier persona que manifieste dificultades en la escuela, y así contribuir al crecimiento y desenvolvimiento de todos: profesores, estudiantes y personal administrativo.

Para Costabile y Brunello (2005), la inclusión es un proceso dinámico que depende de constantes evaluaciones, reflexiones, discusiones con las prácticas y vivencias, para que se torne realmente efectivo. Resalta, que como cualquier propuesta innovadora, ésta no se hace efectiva por decreto, sino que también, por acciones que posibilitan su viabilidad y por la disposición de las personas en aceptar la diversidad como condición inherente a la sociedad. La inclusión es un proceso formado por niveles, presupone por ejemplo, el estar en la escuela, en la medida en que los estudiantes con discapacidad deben estar en el mismo espacio geográfico que los demás estudiantes, traspasando barreras de las antiguas clases especiales; la participación, que significa dar condiciones para que los estudiantes, más allá de ubicarse en el mismo espacio, participen de las actividades; y la adquisición de conocimiento, que sintetiza la razón de existencia de la institución escolar, entre otros. Bartaloti y Carlo (2001) afirman que es fundamental percibir que no basta proporcionar al sujeto situaciones de interacción, una vez que en las relaciones educativas se establece un proceso de mediciones planeadas y que posibilitan el aprendizaje; por eso, también es necesario conocer los caminos recorridos por los individuos, su cultura, su medio social, sus experiencias y así, poder construir una relación de enseñanza y aprendizaje que sea eficiente y que este proceso sea visto como un todo.

Cruz y Emmel (2007) señalan sobre la necesidad de realizar investigaciones en el ambiente escolar, a fin de conocer los tipos de actividades manuales requeridas en este contexto, más allá de las situaciones demandadas por los profesores y colegas de clases, en lo que compete al desempeño ocupacional de niños con discapacidad, así como las adaptaciones ambientales, las actividades preescolares (tareas), la necesidad o no del uso de equipamientos de asistencia que puedan estimular el uso bilateral o mejorar el desempeño funcional de tareas que exigen el uso de las manos en los niños. Ya, Lopes y Silva (2007) describen que experiencias nacionales e internacionales han sido descritas y reveladas a las escuelas como focos de atención frente a la terapia ocupacional. Así, colaborar en la formación de educadores en el sentido del desarrollo de las competencias 
de cada profesional en sus acciones, manteniendo el diálogo sobre los contenidos teóricos y los principios que caracterizan el proyecto político pedagógico, hace de este contexto un espacio de relevancia en la actuación en la Terapia Ocupacional, porque cuando se produce la interacción de un equipo multidisciplinar traen un resultado significativo para la educación inclusiva.

\section{Terapia Ocupacional}

La profesión de la Terapia Ocupacional fue propuesta por George E. Barton, en la primera década del siglo XX en los Estados Unidos de América, con la Asociación Americana de Terapia Ocupacional (2007; 2010) Cavalcanti y Galvão (2007), señalan que la Terapia Ocupacional surgió como una acción profesional precisa, con objetivos, objetos de trabajo, instrumentos y clientes a partir de dos marcos históricos: la Revolución Francesa, en 1789 y la Primera Guerra Mundial, en 1914. Esos dos hechos políticos y económicos marcaron históricamente el siglo XIX, porque exceden la cronología de un siglo, época de una serie de revoluciones contra el orden establecido, o en otros términos, el avance de la democracia como proyecto político de la burguesía en relación a la caída de la aristocracia, el liberalismo económico en cuanto proyecto político, y el racionalismo en cuanto pensamiento hegemónico, además de la superación de las consecuencias de la salud en la Primera Guerra Mundial.

En América Latina, la Terapia Ocupacional surgió principalmente relacionada a las actividades de rehabilitación, contuvo, también fue ligada a las áreas de promoción de la salud y bienestar, incluso en las humanidades, en la segunda década del siglo XX, como por ejemplo, en Brasil, Argentina y Chile, entre otros países. Específicamente en Brasil, la Terapia Ocupacional fue traída por la familia real. También fue llamada ergoterapia, praxiterapia y laborterapia, las cuales gradualmente fueron substituidas. Así la Terapia Ocupacional pasó a ser considerada como el arte y la ciencia de direccionar la respuesta del Hombre a una actividad seleccionada para la realización de las tareas que mantienen, recuperan, reorganizan y desarrollan sus capacidades, motivaciones y estilos de vida necesarios para su inclusión social, porque la Terapia Ocupacional es una profesión compleja, fundamentada en una amplia base de conocimiento tanto de las ciencias de la salud como de las ciencias sociales y además también su práctica, que incorpora habilidades correctivas, técnicas y creativas, con procesos especializados de actuación en todas los grupos etarios y para una gran variedad de problemas físicos, sociales y ambientales.

La Terapia Ocupacional actúa en conjunto con un equipo multidisciplinar, con el objetivo del análisis y aplicación terapéutica de actividades, indicación y/o confección de adaptaciones aplicadas de manera directa o indirecta, física o mental, activa o pasiva, preventiva, correctiva o de adaptación, con el objetivo de prevenir, recuperar, mejorar y promover la salud y la calidad de vida. Para Bartaloti y Cralo, la Terapia Ocupacional actúa sobre los déficits físicos, mentales y sociales, que dificultan o amenazan la funcionalidad de los niños, adultos o adultos mayores, contribuyendo a la no exclusión social, por medio de la mantención del nivel máximo de funcionalidad e independencia.

De este modo, la Terapia Ocupacional se utiliza en actividades humanas como forma de quebrantar acciones exclusivas que impidan el aprendizaje y el acceso a la permanencia de adolescentes y niños en las escuelas, porque la actividad, en cuanto acción significativa y potencializada del acto creativo, sustentada en una relación de confianza, que permite la expresión cultural de cada estudiante, construyendo un campo común, posibilitando la interacción entre los individuos involucrados (Jurdi, 2004).

Rocha describe que históricamente la Terapia Ocupacional en el campo de la educación, se insertó con el nombre de "Educación Especial" orientada para personas con discapacidad, en instituciones educacionales especializadas, segregadas del sistema tradicional de enseñanza, o del trabajo desarrollado en las denominadas "clases especiales" dirigidas a las poblaciones específicas, principalmente a estudiantes con discapacidad intelectual, física, visual, auditiva o de trastorno de desarrollo. En esta perspectiva, la acción de la terapia ocupacional abarca un conjunto de esfuerzos de rehabilitación dirigidos al individuo, con el fin de proporcionar el desarrollo de habilidades y competencias de personas con discapacidad para su adaptación al medio educacional y social. Para Jurdi, Brunello y Honda, el convenio con el campo de la educación ha permitido que la Terapia Ocupacional presente acciones direccionadas para el diario vivir escolar, porque deben ser acompañados de discusiones sobre prácticas más efectivas que contemplen las actividades del diario vivir y las relaciones que se establecen en este contexto.

En Canadá, la consolidación teórica y técnica de la Terapia Ocupacional está relacionada a un gran esfuerzo colectivo, coordinado por la Asociación Cana- 
diense de Terapeutas ocupacionales, como respuesta a presiones sociales y gubernamentales relacionadas a la necesidad de establecer parámetros que pueden medir el impacto y la calidad de los servicios ofrecidos por los terapeutas ocupacionales, y justificar su inserción en el contexto de la reestructuración de los sistemas locales de salud, especialmente para definir acciones financieras por los recursos públicos, una vez que la discusión sobre el control de los gastos y aumento de costos de los servicios de salud, aliada a la presión de los consumidores para mejorar los servicios y control de los cuidados recibidos, juntamente con los cambios en los perfiles epidemiológicos, como por ejemplo, el creciente desplazamiento de los servicios en dirección a la comunidad, ya que fueron factores impulsores de los cambios en la terapia ocupacional, que pasó a ofrecer prácticas que realmente satisficiesen las necesidades de las personas (Law, 1998).

En 1979, se inició un proceso de trabajo que involucró a un grupo de profesionales reconocidos en el área de la terapia ocupacional canadiense, que elaboraron directrices para nortear el conjunto de las prácticas desarrolladas por la terapia ocupacional en el país, ya que la meta era proporcionar un campo común de diálogo y producción teórico-práctico, dado que el área todavía se caracterizaba por una gran dispersión técnica y conceptual, surgiendo muchas prácticas que, casi exclusivamente, se daban en la cultura oral. Como resultado de este esfuerzo, surgió la orientación de la "Terapia Ocupacional Centrada en el Cliente", que inspirada en las formulaciones de Carl Rogers, privilegiaba la calidad de interacción entre terapeuta y cliente en el proceso terapéutico, donde se reconoce al cliente como un sujeto en la construcción de proyectos terapéuticos (Law, 1995). Con todo eso, esta orientación implica una profunda reflexión sobre las cuestiones que son fundamentales en el desarrollo de la Terapia Ocupacional, como la interacción entre terapeuta y cliente y la distribución de poder involucrada en esta relación; el desplazamiento de un tipo de relación terapeuta-cliente muy directiva y marcada por el modelo médico, para una relación donde el terapeuta se coloca como un elemento facilitador; el desplazamiento de un modelo de intervención basado en la disminución de déficits funcionales, para un modelo más preocupado con la habilitación, según metas significativas para el cliente; la importancia del ambiente en los resultados de la intervención que a su vez, pierden de vista la singularidad de las necesidades de cada persona en los más diferentes contextos.
Tales procedimientos facilitan la comprensión del proceso enseñanza-aprendizaje, que aliados al conocimiento sobre el desarrollo humano y su desempeño ocupacional, posibilita al terapeuta ocupacional colocarse como un compañero esencial para el desarrollo de los trabajos en varios espacios, programas y recursos educacionales. De este modo, se puede considerar que la articulación de la Terapia Ocupacional con la educación permite comprender cómo las personas se relacionan con los otros y con los objetivos de conocimiento, porque el terapeuta ocupacional trabaja con la persona en actividad, evaluando el desempeño ocupacional en lo que se refiere a las habilidades para desempeñar tareas, buscando así, incidir en los roles ocupacionales de modo satisfactorio y apropiado al estado de desarroIlo cultural y ambiental del individuo, y de acuerdo con la dinámica institucional.

\section{Desempeño ocupacional}

Para Zanni, Bianchin y Marques (2009), el desempeño ocupacional se refiere a las habilidades de los clientes para seguir y mantener una rutina diaria, desempeñar papeles sociales y tareas que tienen como objetivo la automantención, la producción y la recreación; ejecutadas de modo satisfactorio y apropiado para el nivel de desarrollo, cultura y ambiente del individuo. Se divide en tres componentes: (a) Actividades de la vida diaria o AVD; Actividades instrumentales de la vida diaria o AIVD; y las actividades de diversión o recreación; (b) Componentes sensorio-motores, cognitivos y psicológicos, y de habilidades psicosociales necesarias para el desempeño de las tareas cotidianas; y (c) Contexto del desempeño ocupacional referente a los aspectos temporales y ambientales. El desempeño en estas tres tareas no solo depende de las capacidades de los individuos en los diferentes componentes, sino también, en el medio en que esas actividades son realizadas, en el sentido en que el medio puede colocar exigencias y/o disponer recursos que irán interfiriendo con la realización de las mismas (Magalhaes, 2009).

El desempeño ocupacional engloba la perspectiva del individuo complejo y multidimensional -biológico, psicológico, social y espiritual- que interactúa con su ambiente, utilizando la ocupación para responder de una forma adaptada a las solicitaciones del individuo .

Las tres tareas de desempeño, se dividen a su vez, en tres sub-áreas: (a) Área de Actividades de la Vida Diaria que incluye: los cuidados personales (alimentación, hi- 
giene, vestuario, entre otros); la movilidad (transferencias y movilidad en el interior o exterior); y la gestión en la comunidad (transporte, compra, entre otros); (b) Área de Actividades Productivas que incluye: trabajo (remunerado o voluntario); gestión doméstica (limpieza, tratamiento de las ropas, preparación de colaciones, entre otras); la escolaridad y el juego (en el caso de adecuarse a la edad del sujeto); (c) Área de Actividades de Recreación que incluye: recreación calma (lectura, artesanía); recreación activa (deportes, paseos, viajes); socialización (visitas, telefonemas, correspondencia, entre otras).

En este sentido, el desempeño ocupacional no sólo es influenciado por el rol que el individuo asume en su diario vivir y en su propio ambiente, sino que también, es definido por el individuo en términos de capacidad y competencia para realizar ciertas labores, y en términos de su satisfacción con ese desempeño, porque la esencia de la Terapia Ocupacional se basa en un abordaje integrado y equilibrado de estas tres áreas de desempeño.

En el ámbito de la actuación de la Terapia Ocupacional a través del Modelo de Desempeño Ocupacional, el terapeuta ocupacional busca rehabilitar y/o habilitar la persona para el desempeño de tareas de forma satisfactoria. Como tareas se consideran todas aquellas que el individuo necesita realizar en relación al autocuidado, producción y recreación. El instrumento utilizado se Ilama Medida Canadiense de Desempeño Ocupacional (Canadian Measure of Occupational Performance) - COPM, fundamentada en las Directrices de la terapia Ocupacional para la Práctica Basada en el Cliente y en el Modelo Canadiense de Desempeño Ocupacional. La COPM tiene como uno de sus puntos fuertes un amplio foco en el desempeño ocupacional en todas las áreas de la vida, del autocuidado a la producción, considerada una evaluación individualizada en la forma de entrevista semi-estructurada que mide la auto-percepción del cliente en el desempeño ocupacional (15), porque la evaluación abarca el papel del cliente y sus expectativas, de este modo, que las cuestiones identificadas sean relevantes.

La COPM ha sido traducida y adaptada a los más diversos contextos, exhibiendo su evidente utilidad, comprobada por la extensa bibliografía disponible sobre el tema. Aunque todavía no había sido publicado, el formulario de la COPM fue traducido para el portugués en el final de la década de 1990 y el Manual de instrucción fue preparado en la versión brasilera y publicado en el año 2009. Una de las ventajas de la COPM, que determinó su traducción en diversos países, es que como no existían tareas prefijadas en el ins- trumento, se consigue evaluar al cliente de acuerdo con su propia perspectiva, considerando su desempeño en tareas culturalmente relevantes.

De acuerdo con Carswell, McColl, Baptiste, Law, Polatajko y Pollock, la COPM ya fue traducida a 20 idiomas y es usada en 35 países, y existen evidencias de que posee buena confiabilidad, validez y utilidad clínica para cuantificar los frutos obtenidos en el desempeño ocupacional, como resultado de la intervención terapéutica. Considerando la actual tendencia para la práctica centrada en el cliente, la COPM aparece como recurso simple, de fácil utilización, que permite no sólo focalizar las metas del cliente, sino que también ayuda a presentar la terapia ocupacional, una vez que en el proceso de entrevista queda explícita la función de la terapia.

Law, Baptiste, Mccoll, Polatajko y Pollock, describen que la traducción de la COPM para diversos idiomas ha sido acompañada de proyectos de investigación que buscan identificar su validez en diferentes contextos, porque investigadores hicieron observaciones sobre la responsabilidad frente a los cambios, facilidad de administración, tiempo para la conclusión y habilidad para comunicar aspectos de la ocupación. Observaron que la COPM ofreció beneficios significativos en una amplia variedad de ambientes clínicos con diferentes poblaciones y en diferentes idiomas y cultura. Considerando la práctica clínica, Parker y Sykes (2006) apuntan como aspectos positivos de la COPM su sensibilidad para medir el resultado de una variedad de intervenciones de la terapia ocupacional, más allá del hecho de que el instrumento es útil para clientes de diferentes ambientes y discapacidades/incapacidades, y especialmente por ser una medida independiente de diagnósticos específicos. La COPM ofrece una estructura útil para la evaluación inicial de todas las áreas de desempeño ocupacional, y ayuda a implementar un abordaje centrado en el cliente, ya que durante el proceso de evaluación, las propiedades del cliente se tornan evidentes, y estas generalmente son diferentes de las ideas iniciales del terapeuta (Mew MM, Fossey E, 1996).

Con base en lo expuesto, se puede determinar que la posibilidad de una educación de calidad para las personas con discapacidad es una necesidad ética y legal en la actualidad, una vez que el censo escolar viene demostrando un creciente aumento en matrículas de personas con discapacidades en las escuelas tradicionales de sistemas educativos en prácticamente todos los países que ofrecen la inclusión. En Brasil, de acuerdo con el MEC/INEP (Ministerio de Educación de Brasil, 2009), existen casi 700.000 estudiantes con dis- 
capacidad matriculados; de ese total más de la mitad estudian en escuelas regulares, un tercio en escuelas especializadas, y poco más un décimo de estas personas frecuentan clases especiales en escuela común. En este sentido, si por un lado, la terapia ocupacional viene a contribuir con la inclusión, por otro lado, se destaca la necesidad de ofrecer una escuela que esté preparada para recibir personas con discapacidad como ellos son, a través de un ambiente inclusivo, donde se pueda acoger a todos sin excepción (Mantoan, 2001).

\section{CONSIDERACIONES FINALES}

Al discutir sobre los aspectos relacionados al desempeño ocupacional de personas con discapacidad en la educación inclusiva, a través de una revisión teórica, se observa que la COPM ha sido traducida y adaptada a los más diversos contextos, exhibiendo una evidente utilidad comprobada por la extensa bibliografía disponible sobre el tema, y presenta como punto fuerte, un amplio foco en el desempeño ocupacional en todas las áreas de la vida, del autocuidado a la producción, porque la esencia de la terapia ocupacional se centra en un abordaje integrado y equilibrado en estas tres áreas de desempeño, y todavía puede ser considerada como una evaluación individualizada y con forma de entrevista semi-estructurada que mide la autopercepción del cliente en el desempeño ocupacional, más allá de ofrecer subsidios al mejor desempeño y la satisfacción de las actividades seleccionadas. Así, la actuación del terapeuta ocupacional, junto con las personas con discapacidad en la educación inclusiva, más allá de contribuir significativamente al desarrollo de satisfactorios papeles ocupacionales, a través de nuevas posibilidades ocupacionales, abre un abanico de posibilidades en la actuación del terapeuta ocupacional en este contexto.

\section{ReferenCias Bibliográficas}

Almeida MC., CAMPOS GWS. Políticas e modelos assistenciais em saúde e reabilitação de pessoas com deficiência no Brasil: análise de proposições desenvolvidas nas últimas duas décadas. Revista de Terapia Ocupacional da Universidade de São Paulo. 2002; 13(3): 118-126.

Araújo LS., Jordán NF., Monclus PG., O. Ciencia de la ocupación y terapia ocupacional: sus relaciones y aplicaciones a la práctica clínica. Revista Chilena de Terapia Ocupacional. 2011; 11(1): 79-87.

Bartalotti CC., Carlo MMRP. (Orgs.). Terapia ocupacional no Brasil: fundamentos e perspectivas. São Paulo: Plexus, 2001.

Bartalotti CC., Carlo MMRP. (Orgs.). "Terapia ocupacional no Brasil: fundamentos e perspectivas". São Paulo: Plexus, 2001.

Bartalotti CC., Carlo MMRP. Terapia ocupacional no Brasil: fundamentos e perspectivas. São Paulo: Plexus, 2001.

Brasil. Constituição do. Brasília: Senado Federal, Centro Gráfico, 1988.

Brasil. Lei de Diretrizes e Bases da Educação: Lei 9.394/96. Diário Oficial da União, Brasília, de 23 de dezembro de 1996.

Brasil. Ministério da Educação. Instituto Nacional de Estudos e Pesquisas Educacionais Anísio Teixeira. Sinopse estatística da educação básica. Brasília: INEP, 2009.

Caot, Canadian Association of Occupational Therapists. Occupational therapy guidelines for client centred practice. Publications ACE, Toronto, 1991.

Carpenter L., Bacer G., Tyldesley B. The use of the Canadian occupational performance measure as an outcome of a pain management program. 2001; 68(1): 16-22.

Carswell A., Mccoll MA., Baptiste S., LAW M., Polatajko H., Pollock $\mathrm{N}$. The Canadian Occupational Performance Measure: a research and clinical update. Canadian Journal of Occupational Therapy. 2004; 71(4): 16-22.

Cavalcanti A., Galvão C. Terapia Ocupacional: Fundamentação e Prática. Rio de Janeiro, Koogan, 2007.

Chalita, G., Educação: A solução está no afeto. São Paulo: Gente, 2004.

Corrêa RM. Dificuldades no aprender: um outro modo de olhar. Campinas: Mercado das Letras, 2001.

Costabile C., Brunello MIB. Repercussões da inclusão escolar sobre o cotidiano de crianças com deficiência: um estudo a partir do relato das famílias. Revista de Terapia Ocupacional da Universidade de São Paulo. 2005; 16(3): 124-130.

Cruz CMD., Emmel GLM. O brinquedo e o brincar na estimulação da função manual de crianças pré-escolares com deficiência física. Cadernos de Terapia Ocupacional da UFSCar. 2007; 15(1): 7-17.

Jurdi APS., Brunello MIB., Honda M. Terapia ocupacional e propostas de intervenção na rede pública de ensino. Revista de Terapia Ocupacional da Universidade de São Paulo. 2004; 15(1): 26-32.

Law M., Baptiste S., Carswell A., Mccoll MA., Polatajko H., Pollock N. Medida Canadense de Desempenho Ocupacional (COPM). Belo Horizonte: Editora UFMG, 2009.

Law M., Baptiste S., Carswell A., Mccoll MA., Polatajko H., Pollock N. Canadian Occupational Performance Measure (COPM). Ottawa: CAOT Publications, 2000. 
Law M., Baptiste S., Mills J. Client-centred practice: what does it mean and does make a difference? Canadian Journal of Occupational Therapy. 1995; 62(5): 250-256.

Law M. Client-centered occupational therapy. New York: Slack, 1998.

Lillo SG., Blanche, El. Desarrollo de la terapia ocupacional en Latinoamérica. Revista Chilena de Terapia Ocupacional. 2010; 10: 123-135.

Lopes RE., Silva CR. O campo da educação e demandas para a terapia ocupacional no Brasil. Revista de Terapia Ocupacional da Universidade de São Paulo. 2007; 18(3): 158-164.

Lorenço GF., CID MFB. "Possibilidades de Ação do Terapeuta Ocupacional na Educação Inclusiva". Cadernos de Terapia Ocupacional da UFSCar. 2010; 18(2): 169-179.

Magalhães LV., Cardoso AA., Magalhães LC. Medida Canadense de Desempenho Ocupacional (COPM). Belo Horizonte: Editora UFMG, 2009.

Mângia EF. Terapia Ocupacional: práticas, discursos e a questão da legitimidade científica. Revista de Terapia Ocupacional da Universidade de São Paulo. 1999; 10(2-3): 60-68.

Mantoan MTE. Caminhos pedagógicos da inclusão. São Paulo: Memnon, 2001.

Mantoan MTE. Educação escolar de deficientes mentais: Problemas para a pesquisa e o desenvolvimento. Cadernos Cedes. 1998; 46: 93-107.

Mantoan MTE. Inclusão escolar: caminhos e descaminhos, desafios, perspectivas. Ensaios Pedagógicos. Brasília: Ministério da Educação, 2006.

Marins S. Escola inclusiva. São Carlos: Edufscar, 2002.

Mendes EG. "A radicalização do debate sobre inclusão". Revista Brasileira de Educação. 2006; 11(13): 387-404.

Mendes EG. "Perspectivas para a construção da escola inclusiva". En PALHARES MS, MARINS S. "Escola inclusiva". São Carlos: Edufscar, 2002, p. 61- 86.

Mendes EG. Perspectivas para a construção da escola inclusiva. En PALHARES MS,

Mew MM., Fossey E. Client aspects of clinical reasoning during na initial assessment using the Canadian Occupational Performance Measure. Australian Journal of Occupational Trerapy. 1996; 43(3-4): 155-166.

Oliveira C., Castanharo RCT. O terapeuta ocupacional como facilitador do processo educacional de crianças com dificuldades de aprendizagem. Cadernos de Terapia Ocupacional da UFSCar. 2008; 16(2): 91-99.

Parker D., Sykes CH. A Systematic Review of the Canadian Occupational Performance Measure: a clinical practice perspective. British Journal of Occupational Therapy. 2006; 69(4): 150-160.

Parker D., Sykes CH. A Systematic Review of the Canadian Occupational Performance Measure: a clinical practice perspective. British Journal of Occupational Therapy. 2006; 69(4): 150-160.
Rebeiro K. Client perspectives on occupational therapy practice: are we truly client-centered? Canadian Journal of Occupational Therapy. 2000; 67(1): 2000.

Rocha EF., LUIZ A., ZULIAN MA., R. Reflexões sobre as possíveis contribuições da terapia ocupacional nos processos de inclusão escolar. Revista de Terapia Ocupacional da Universidade de São Paulo. 2003; 14(2): 72-78.

Rocha EF., LUIZ A., ZULIAN MAR. Reflexões sobre as possíveis contribuições da terapia ocupacional nos processos de inclusão escolar. Revista de Terapia Ocupacional da Universidade de São Paulo. 2003; 14(2): 72-78.

Rocha EF. "A Terapia Ocupacional e as ações na educação: aprofundando interfaces". Revista de Terapia Ocupacional da Universidade de São Paulo. 2007; 18(3): 122-127.

Sanches, Isabel e TEODORO, António. Da integração à inclusão escolar: cruzando perspectivas e conceitos. Revista Lusófona de Educação. 2006; (8): 63-83.

Santos MP. Educação inclusiva e a declaração de Salamanca: consequências ao sistema educacional brasileiro. Revista Integração. 2000; 22: 34-40.

Sumsion T. Prática baseada no cliente na terapia ocupacional: guia para implementação. São Paulo: Roca, 2003.

Testa D. Aportes para el debate sobre los inicios de la profesionalización de la terapia ocupacional en Argentina. Revista Chilena de Terapia Ocupacional. 2012; 12(1): 72-87.

Urdi APS., Brunello MIB., Honda M. Terapia ocupacional e propostas de intervenção na rede pública de ensino. Revista de Terapia Ocupacional da Universidade de São Paulo; 15(1): 26-32.

Zanni KP., Bianchin MA., Marques LHN. Qualidade de Vida e Desempenho Ocupacional de Pacientes Submetidos à Cirurgia de Epilepsia. Journal of Epilepsy and Clinical Neurophysiology. 2009; 15(3): 114-117. 